\title{
AUTOMATIC REGISTRATION OF GF4 PMS: A HIGH RESOLUTION MULTI- SPECTRAL SENSOR ON BOARD A SATELLITE ON GEOSTATIONARY ORBIT
}

\author{
Min Gao ${ }^{1,2 *}$, Junsheng $\mathrm{Li}^{2}$ \\ ${ }^{1}$ Capital Normal University, Beijing 100048, China - 244061677@qq.com \\ ${ }^{2}$ Key Laboratory of Digital Earth Science, Institute of Remote Sensing and Digital Earth, Chinese Academy of Sciences, Beijing \\ 100094, China-lijs@ radi.ac.cn
}

Commission III, ICWG III/Ivb

KEY WORDS: GF4 PMS, Automatic Image Registration, Optimal Base Band, RPC Correction, Spatial Resolution

\begin{abstract}
:
Geometric correction is an important preprocessing process in the application of GF4 PMS image. The method of geometric correction that is based on the manual selection of geometric control points is time-consuming and laborious. The more common method, based on a reference image, is automatic image registration. This method involves several steps and parameters. For the multi-spectral sensor GF4 PMS, it is necessary for us to identify the best combination of parameters and steps. This study mainly focuses on the following issues: necessity of Rational Polynomial Coefficients (RPC) correction before automatic registration, base band in the automatic registration and configuration of GF4 PMS spatial resolution.
\end{abstract}

\section{INTRODUCTION}

The first high-resolution remote-sensing satellite GF4 was launched successfully on a geosynchronous orbit on December 29, 2015.GF4 carries a panchromatic multispectral camera (Panchromatic and Multispectral Scanner, PMS) and an infrared camera (Infrared Scanner, IRS). GF4 PMS has a panchromatic band $(0.45 \sim 0.90 \mu \mathrm{m})$ and four multispectral bands, namely, blue band $(0.45 \sim 0.52 \mu \mathrm{m})$, green band $(0.52 \sim 0.60 \mu \mathrm{m})$, red band $(0.63 \sim 0.69 \mu \mathrm{m})$, and an infrared band $(0.76 \sim 0.90 \mu \mathrm{m})$. Their spatial resolution is $50 \mathrm{~m}$, which is highest among remote sensing satellites on the geosynchronous orbit. Owing to the high temporal resolution and high spatial resolution, GF4 PMS plays a vital role in the monitoring of the environment, disasters and surface resources ( $\mathrm{Li}, 2016)$.

Geometric correction is an important preprocessing process in the application of GF4 PMS image. (Yu \& Tian, 2017)The method of geometric correction that is based on the manual selection of geometric control points is time-consuming and laborious. The more common method, based on a reference image, is automatic image registration. This method involves several steps and parameters. For the multi-spectral sensor GF4 PMS, it is necessary for us to identify the best combination of parameters and steps. This study mainly focuses on the following issues:

1. Necessity of Rational Polynomial Coefficients (RPC) correction before automatic registration: GF4 PMS provides the parameters for RPC correction, and we may or may not use these parameters to cause the RPC correction before automatic registration. (Liu,2013) Therefore, it is important to ensure that RPC correction improves the accuracy of automatic registration.
2. Base band in the automatic registration: We need to choose a base band in the automatic registration process. Different application scenes have different optimal bands. We must be aware of the optimal band being applied to the large lake area during automatic registration.

3. Configuration of GF4 PMS spatial resolution: GF4 PMS is a geosynchronous satellite, the sub-star point of which is on the equator with a spatial resolution of $50 \mathrm{~m}$. Higher latitude, lowers the actual spatial resolution. The resample pixel size needs to be considered as the resolution of the sub-star point or the actual spatial resolution.

\section{DATA AND METHOD}

\subsection{Data}

The initial data consist of six GF4 PMS images of Taihu Lake. These images were captured on July 22, July 23, July 24, August 18, August 24, and August 29 in 2016, respectively. The reference images used are Landsat8 OLI images that have undergone geometric precision correction. The two Landsat8 OLI images that we chose as base images for the two sets of three GF4 PMS images in July and August were captured on July 27, 2016 and August 18, 2016, respectively.

\subsection{Automatic Registration Method}

We use Image Registration Workflow of ENVI5.3 (The Environment of Visualizing Images) to perform automatic registration. This method utilizes regional grey, which compares the difference between similar sized grey levels in the two images and judges the range and position of the overlapped region by the difference (Yang Xuedong, 2013).

\footnotetext{
* Corresponding author
} 


\section{PROCESS AND PARAMETERS CONFIGURATION}

To verify if RPC correction is essential, the original GF4 PMS images and the RPC corrected images were used as input in the Image Registration Workflow. Finally, we compared the results We used the green, red, and infrared bands of GF4 PMS images as base bands to arrive at the optimal band.

The spatial resolution of the Landsat8 OLI reference images is $30 \mathrm{~m}$. These images need to be resampled to the appropriate resolution before they can be used as reference images. We resampled Landsat8 OLI as $50 \mathrm{~m}$ (sub-star point) and $60 \mathrm{~m}$ (near Taihu Lake) to compare the results.

\subsection{Evaluation Method of Registration Accuracy}

Root-Mean-Square (RMS) is the evaluation of the registration effect. RMS may be used to evaluate the necessity of RPC correction preprocessing, the optimal base band, and the most suitable spatial resolution of resampling reference images. This paper used clarity to further evaluate the most suitable spatial resolution of resampling reference images (Wang, 2015).

\section{RESULTS}

Using the methods mentioned above, we made automatical registration to the six GF4 PMS images of Taihu Lake using the resampling Landsat 8 OLI as the reference image. The following results were obtained:

1. We compared the manual selection of control points and the Image Registration Workflow. The number of control points in both is 31 . The workflow lasts for 16 seconds with RMS equal to 0.4. However, manual selection lasts for 38 minutes with RMS equal to 0.7. The part of the resultant images are as shown in Figure 1 and Figure 2. The RMS of the workflow is smaller than that of manual selection. More importantly, there is a significant reduction in the time.

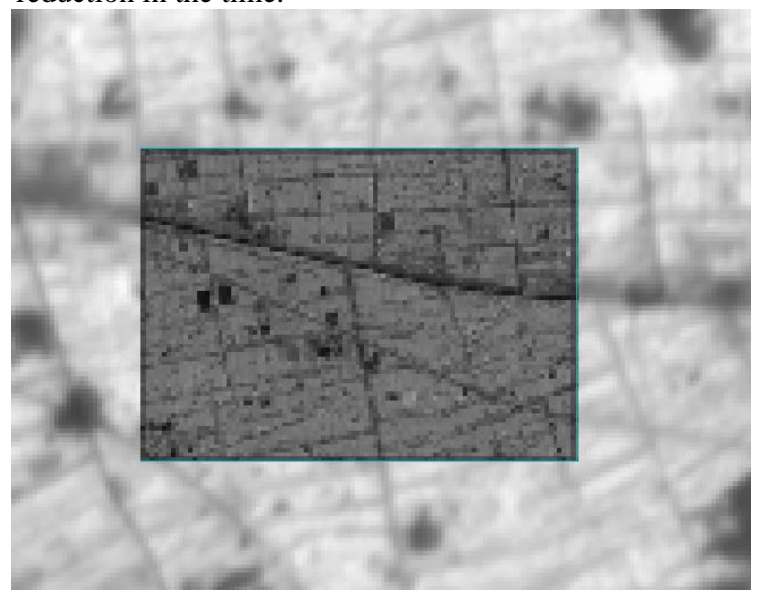

Figure 1. The image registration

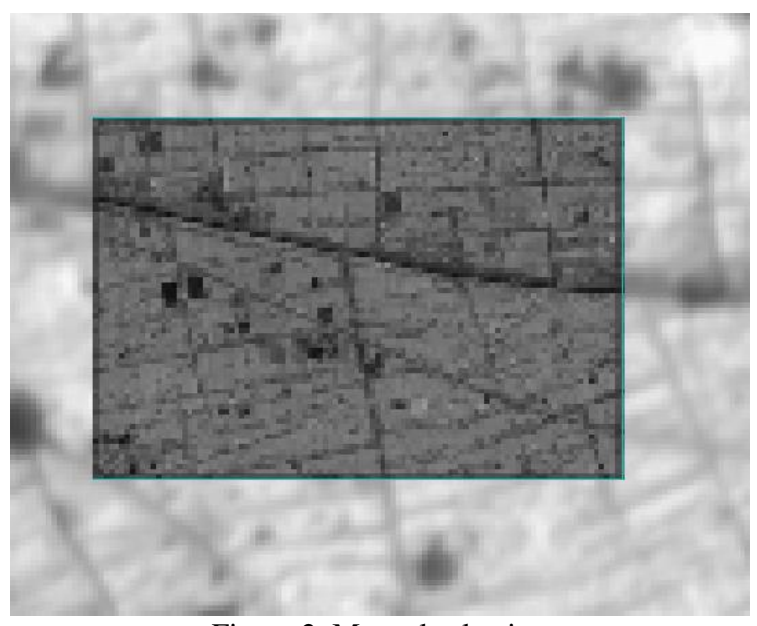

Figure 2. Manual selection

2. Without RPC correction, the average time of registration is 49 seconds and average RMS is 1.3 . On the other hand, with RPC correction, the average time is 13 seconds and the average RMS is 0.4 . We could clearly compare the differences that RPC correction brought in Figure 3, Figure 4, Figure 5 and Figure 6. RPC correction reduces the RMS and processing time. Therefore, RPC correction is necessary before automatic registration.

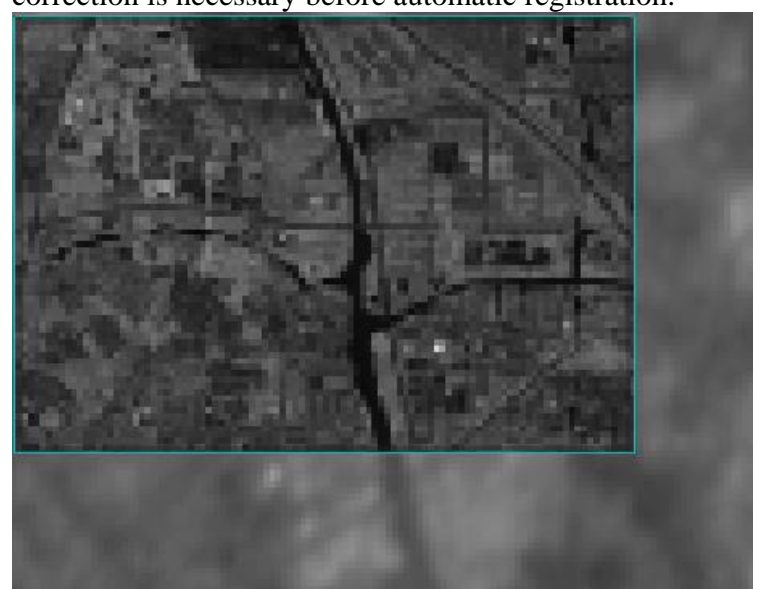

Figure 3. With RPC correction of July 23

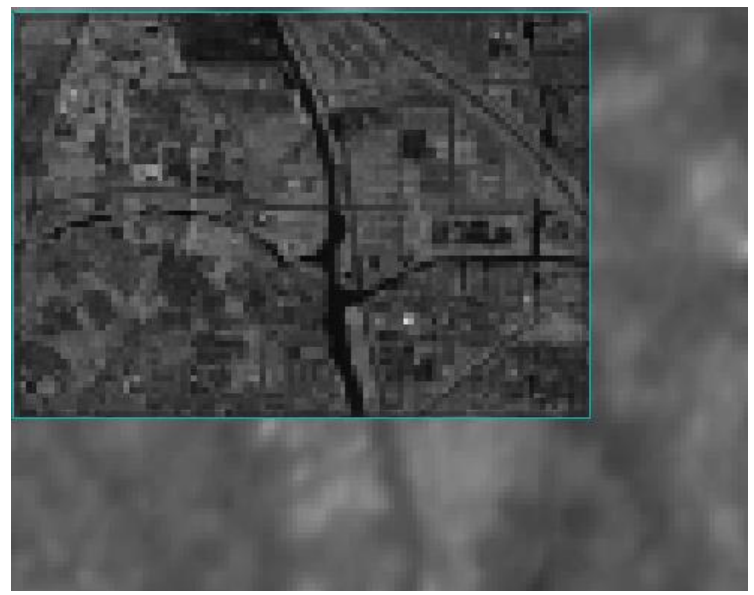

Figure 4. Without RPC correction of July 23 


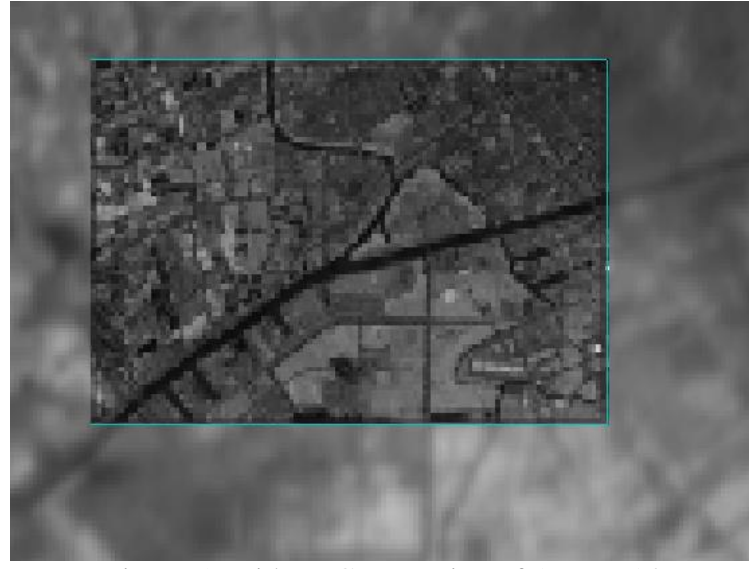

Figure 5. With RPC correction of August 14

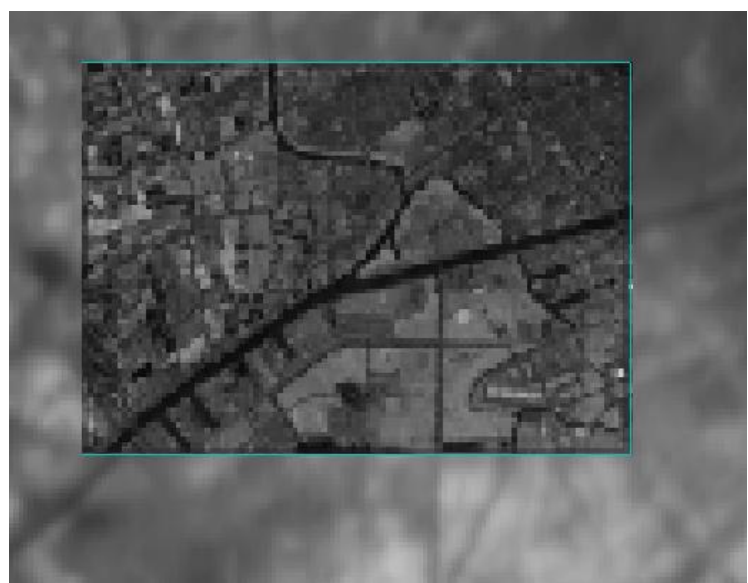

Figure 6. Without RPC correction of August 14

3. When using the red or green bands as the base band, the workflow lasts for 240 seconds on an average, and the average RMS is 0.5. In addition, the GF4 PMS image on August 14 could not produce enough control points. The infrared band lasts for 13 seconds with the average RMS as 0.4 . As a result, the infrared band is the optimal base band.

4. Resampling the Landsat8 OLI image to $50 \mathrm{~m}$ as the base image, the Clarity of the resultant GF4 PMS image is 9. The Clarity of $60 \mathrm{~m}$ GF4 PMS image is 11 . When the base images overlaps the resultant images, we can found the $60 \mathrm{~m}$ images are corrected better. (Figure 7, Figure 8, Figure 9 and Figure 10.) Consequently, resampling the base image to the actual spatial resolution is more effective.

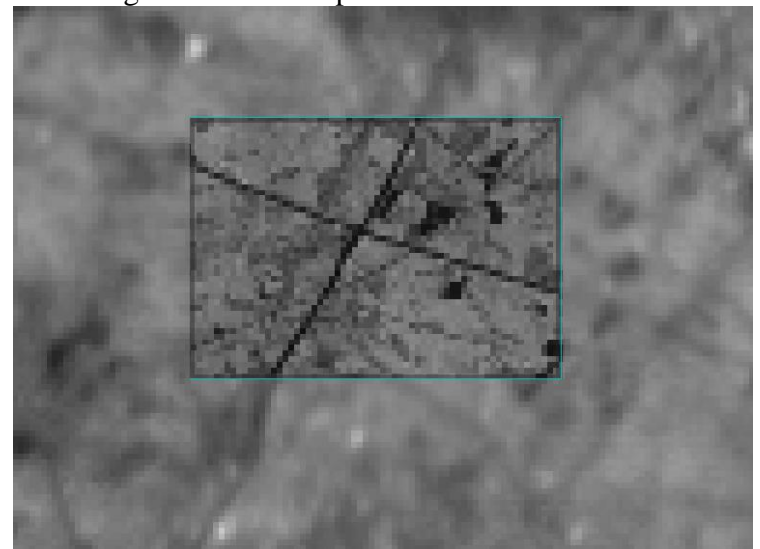

Figure 7. $60 \mathrm{~m}$ resultant images of July 24

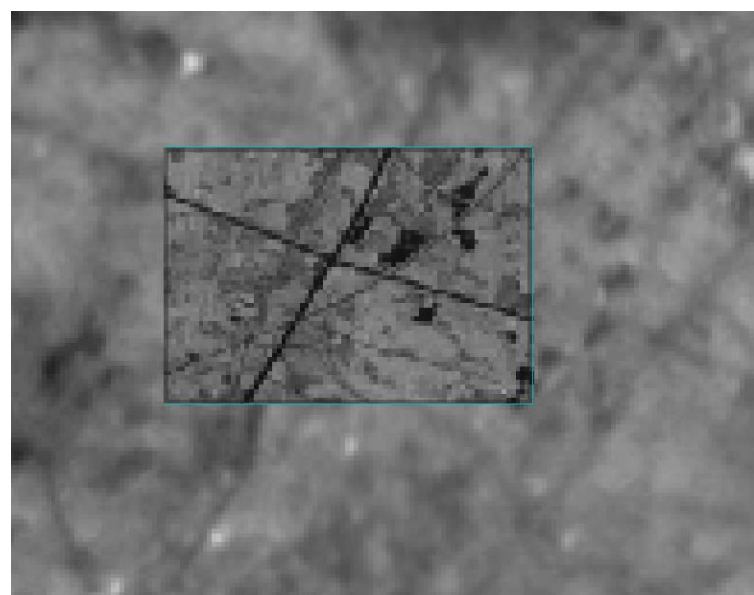

Figure $8.50 \mathrm{~m}$ resultant images of July 24

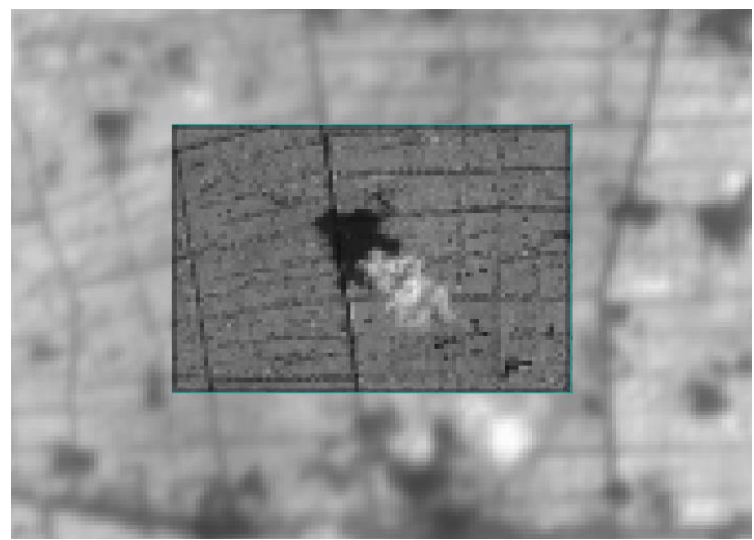

Figure 9. $60 \mathrm{~m}$ resultant images of August 29

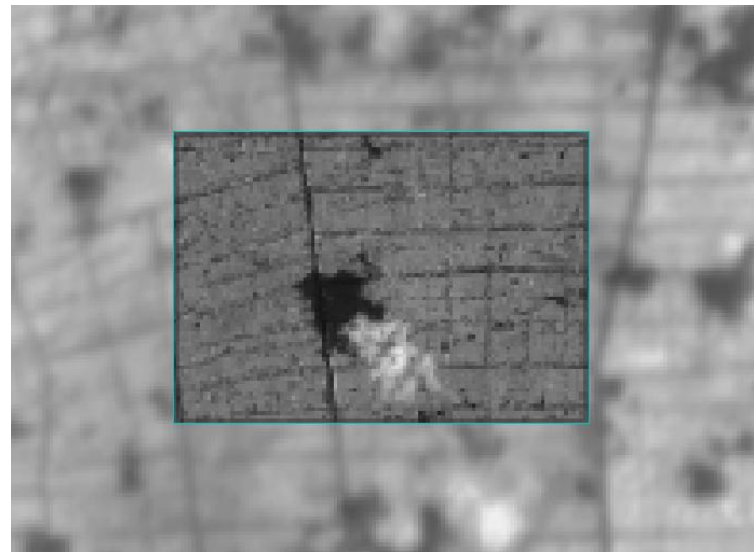

Figure 10. $50 \mathrm{~m}$ resultant images of August 29

\section{CONCLUSIONS}

This paper studies the best solution to the questions mentioned above based on six GF4 PMS images of Taihu Lake. The reference images are Landsat8 OLI images obtained after geometric precision correction. The following conclusions are obtained:

1. The feasibility of automatic registration is high, and it is capable of replacing the manual selection of control point registration.

2. The RPC correction before automatic registration is necessary. 
The International Archives of the Photogrammetry, Remote Sensing and Spatial Information Sciences, Volume XLII-3, 2018 ISPRS TC III Mid-term Symposium "Developments, Technologies and Applications in Remote Sensing", 7-10 May, Beijing, China

3. The infrared band is the optimal base band.

4. Resampling the reference image to the actual spatial resolution gets better results.

\section{REFERENCES}

Li Guo, Kong Xianghao, Liu Fengjing, Lian Minlong, 2016.

GF-4 Satellite Remote Sensing Technology Innovation.

Spacecraft Recovery \& Remote Sensing, 37(4), pp. 7-15.

Liu Jiang, Yue Qingxing, QiuZhenge, 2013. Research on the Approach to RPC Emendation. Remote Sensing for Land \& Resources, 25(1), pp.61-65.

Wang Yuan, Ye Sijing, Yue Yanli, Liu Diyou, Xiong Quan, Zhu Dehai, 2015. Contrast of Automatic Geometric

Registration Algorithms for GF-1 Remote Sensing Image. Journal of Agricultural Machinery, 46, pp. 260-266.

Yu Bowen, Tian Shufang, 2017. Geometric Correction Research on Satellite GF-1 Data. Remote Sensing Technology and Application, 32(1), pp.133-139.

Yang Xuedong, 2013. Automatic Registration of Spot5 Image Based on ENVI4.5. Inner Mongolia Forestry Investigation and Design, 36(6), pp. 124-126. 